\title{
Du Poyet chez Du Fail. Échos de l'Ordonnance de Villers-Cotterêts dans les Contes et discours d'Eutrapel
}

Valerio Cordiner

\section{(2) OpenEdition \\ Journals}

Édition électronique

URL : http://journals.openedition.org/rief/773

DOI : $10.4000 /$ rief. 773

ISSN : 2240-7456

Éditeur

Seminario di filologia francese

Référence électronique

Valerio Cordiner, «Du Poyet chez Du Fail. Échos de l'Ordonnance de Villers-Cotterêts dans les Contes et discours d'Eutrapel », Revue italienne d'études françaises [En ligne], 2 | 2012, mis en ligne le 15 décembre 2012, consulté le 19 avril 2019. URL : http://journals.openedition.org/rief/773 ; DOI : $10.4000 /$ rief. 773

Ce document a été généré automatiquement le 19 avril 2019

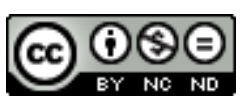

Les contenus de la RIEF sont mis à disposition selon les termes de la Licence Creative Commons Attribution - Pas d'Utilisation Commerciale - Pas de Modification 4.0 International. 


\title{
Du Poyet chez Du Fail. Échos de l'Ordonnance de Villers-Cotterêts dans les Contes et discours d'Eutrapel
}

\author{
Valerio Cordiner
}

\begin{abstract}
À M. Jean-Claude Arnould, humaniste rouennais
\end{abstract}

1 C'est proprement à la lettre qu'il faut prendre, à l'endroit de Du Fail, l'avis de Christian Biet, pour qui «pendant fort longtemps, la littérature s'est appuyée, pour produire et composer ses fictions, sur les procédures juridiques, a emprunté les règles du droit pour élaborer ses intrigues $»^{1}$.

2 En effet, avant d'être le petit humaniste de province écrivant ses bouquins campagnards «aux heures ou les autres coustumierement s'escurent les dents $»^{2}$, le seigneur de la Hérissaye, gentilhomme rennais de vieille souche, marié à une riche héritière des alentours, était déjà "attaché à une tant grave et solide profession »" à savoir la magistrature; une profession qu'il exerça pendant toute sa vie en montant de charge en office jusqu'à la Grande Chambre du Parlement de Bretagne ${ }^{4}$. Assuré à la fois et de l'argent de sa femme et du soutien de M. de Rohan - "hault et puissant $»^{5}$ plénipotentiaire du parti de la France en terre armoricaine - sa carrière brillante dans l'administration judiciaire est aussi marquée par la publication, en 1579, d'un imposant recueil jurisprudentiel composé au profit des rédacteurs de la Nouvelle Coutume.

Or, en plus des Plus notables et solemnels arrests du Parlement de Bretagne et du riche paratexte qui les annote, les Contes et discours d'Eutrapel ${ }^{6}$ sont nourris de fond en comble de doctrine juridique. Le Studium iuris et la pratique du for y dicte et les exemples et la glose explicative, Polygame, Eutrapel et Lupolde étant, à l'instar de leur créateur, des hommes de loi passionnés par les grandes et petites questions relatives à l'administration de la justice. De plus, si l'écriture dufailienne, comme celle de nombreux écrivains de 
temps, se modèle sur le style de chancellerie - un fait dont témoigne le recours systématique au redoublement synonymique ${ }^{7}$ - la matière juridique a un rôle de première envergure dans l'évolution générique du récit dans le dernier quart du XVI ${ }^{e}$ siècle. Avec d'autres arguments doctrinaux, elle contribue en effet au processus général de mutation générique qui intéresse les formes narratives à l'époque des Guerres civiles; et ce, autant chez les conteurs tragiques que chez les auteurs de mélanges bigarrés ${ }^{8}$. En ce qui concerne Du Fail, quoique la narration la plus étendue du recueil, celle des seigneurs de Rivière et de Launay ( $C D E, \mathrm{I}, 43-49)$, se termine sur un procès pénal, le discours juridique, comme tout raisonnement idéologique, agit d'ordinaire sur la matière fictionnelle en tant que vecteur de simplification et souvent de réduction du narratus au rôle de démonstration de la théorie exposée.

Sans revenir sur ce remarquable phénomène littéraire auquel nous avons déjà consacré de plus amples développements 9 , c'est en revanche sous le seul biais de la doctrine juridique (notamment en ce qui concerne les retentissements de la réforme générale de la procédure pénale), que, faute d'espace et dans un souci de cohérence interne, le dernier ouvrage dufailien sera ici analysé.

5 La prémisse à tout raisonnement dufailien autour de la justice est que l'administration de la justice aussi bien que les autres «polices violentes» (CDE, II, 205) doivent échoir aux seuls gentilshommes ( $C D E, \mathrm{I}, 35)$. Fief et justice n'étant en Bretagne qu'une seule et même chose, comme l'idéologie nobiliaire le prescrit et l'infrastructure féodale le requiert, Du Fail soutient que la justice, pour être efficace, doit être administrée sur place, ainsi que le faisaient «pour l'exemple et la terreur du peuple » les anciens rois de France, et le juge sénéchal dans la Péninsule armoricaine ( $C D E, \mathrm{I}, 115-116)$. Utilitaire et expéditive, la justice seigneuriale - y compris celle du roi, le premier gentilhomme de France ${ }^{10}$ - sera donc équitable, c'est-à-dire implacable à l'endroit des coupables. Le copieux inventaire de châtiments et supplices exhibé tout le long du recueil en est un solide témoignage ${ }^{11}$.

Ce vœu ne traduit pas seulement le regret de l'âge d'or de la domination féodale. Il est conçu comme motion d'ordre pragmatique en vue de la renaissance morale et matérielle de la gentilhommerie poursuivie par Du Fail. Alors que, de l'avis de l'auteur des Contes et discours, l'« ignoran[c]e des bonnes lettres » (CDE, I, 43) a été l'occasion pour les robins issus de la roture d'assumer de nombreuses charges dans l'administration de la justice, cette transition généralisée des offices judiciaires, dont Du Fail signale à chaque pas les séquelles funestes, dérive en réalité de l'introduction des charges vénales. La vénalité des offices - « si ouverte qu'ayant de l'argent on passoit par tout » (CDE, I, 153) - a engendré une pléthore d'officiers, ainsi qu'une multiplication des procès. Sur l'augmentation des affaires en cours prospère la corruption judiciaire qui désole la France (CDE, I, 29). La pratique judiciaire semble être atteinte, dans son ensemble, par la fièvre de l'argent, sévissant partout sous forme de "faveurs, larrecins, concussions, pilleries » (CDE, II, 275). Du procureur du roi changeant d'avis à la vue d'une pièce d'or (CDE, I, 53), au président de cour souveraine qui admoneste son corrupteur tout en encaissant le dessous-de-table ( $C D E, \mathrm{I}, 56)$, des « couratiers du procés » soulageant les bourses des parties aux sergents et messieurs avaricieux et prévaricateurs (CDE, I, 181), le personnel judiciaire est employé à plein temps à « fouette[r], traine[r], et galoppe[r] la justice à toutes mains» $\left(C D E\right.$, II, 43) ${ }^{12}$. Et, mieux que quiconque, les avocats et chicaneurs, professionnels versés en toute sorte d'ergoterie (CDE, II, 283), adroits manipulateurs des technicismes judiciaires les plus raffinés et malhonnêtes (CDE, I, 8). 
7 « De là », se plaint Du Fail, « est survenu la desolation aux bonnes maisons, la ruine des anciennes races et familles », qui sont les proies les plus recherchées des robins. C'est la motivation pour laquelle il sollicite du roi, à qui cela échoit en tant que premier magistrat du royaume (CDE, I, 119), une « bonne et saincte reformation de tous estats » $(C D E, I, 29)$, conçue à l'effet de restituer la potestas iudicandi aux mains de ses légitimes détenteurs, à savoir les nobles de sang (CDE, II, 276). Or, la principale réforme de la justice ayant eu lieu au cours du XVI ${ }^{\mathrm{e}}$ siècle est précisément cette

ordonnance de l'an 1539, laquelle les maistres chiquaneurs n'ont jamais voulu recevoir, l'appellans Guillemine, pour avoir esté faite par maistre Guillaume Poiet, lors chancelier de France; ce qui certainement chasseroit les pigeons du colombier et couperoit la racine d'une infinité de procés (CDE, I, 36)

Un jugement flatteur que celui de Du Fail qui, en plus d'autres références sur lesquelles on reviendra par la suite, s'étend aussi à la personne de son rédacteur : ce Guillaume Poyet dont Du Fail regrette qu'il n'ait pas été chancelier de France "en ces derniers troubles, aussi bien qu'il estoit l'an mil cinq cens trente neuf, lors qu'il rongna les ongles de si prés à la puissance et jurisdiction ecclesiastique » (CDE, I, 54).

Il y a vraiment de quoi s'étonner de cet éloge répété, mis dans la bouche d'Eutrapel et de Polygame, défendant partout ailleurs les attributs de la noblesse d'épée. Fils d'un petit avocat angevin, Guillaume Poyet est de son temps le champion inégalé du carriérisme bourgeois. Grâce à ses talents oratoires et à son absence de scrupules, moyennant finances et à l'ombre des puissants, il sut arriver d'échelon en échelon au faîte de la hiérarchie judiciaire. Praticien chevronné du droit, promu avocat du roi, puis membre du Grand Conseil, président à mortier du Parlement de Paris et finalement chancelier de France, il fut aussi une sorte de surintendant des finances lâchant la bride de la vénalité des charges d'une manière inaccoutumée et avec un avantage personnel certain. Autoritaire, cupide et vindicatif, il finit par perdre, avec l'appui de la cour et du Parlement, et son prestige et ses richesses. Inculpé, lors d'un procès engagé contre lui à l'initiative du roi (1542-1545), d'assommants chefs d'accusation - malversation, péculat, faux et usage du faux, abus de pouvoir, concussion, création et disposition d'offices, etc. il fut aussi la victime des mesures vexatoires pour les accusés, contenues aux articles 154 et 162 de cette même ordonnance qui lui assura une renommée durable.

Convaincu de ces crimes énormes, il fut condamné à l'interdiction perpétuelle de toute fonction publique, à cent mille livres d'amende, à cinq ans de prison et finalement au blâme unanime des contemporains ${ }^{13}$, entre autres Pierre Ayrault, Jean Constantin et Charles Du Moulin, dont on connaît le verdict impitoyable : «Vide tyrannicam opinionem illius impii Poyeti [...]. Vide duritiem iniquissimam per quam etiam defensio aufertur ${ }^{14}$; et, à leur suite, les chicanous dufailiens contrariés dans leurs manèges, des historiens et philosophes férus d'anglophilie ${ }^{15}$, les tenants du système accusatoire et de la présomption d'innocence.

11 C'est peut-être trop, avouons-le, toute cette hostilité pour un juriste et un ministre qui fut avant tout un homme d'État (quoique peu scrupuleux), dont le dessein fut simplement de favoriser la centralisation in fieri des pouvoirs, en remédiant de son mieux aux lenteurs et aux manques d'efficacité de la justice; et qui, de surcroît, sut introduire, toujours sous l'égide de l'absolutisme, d'autres innovations remarquables dans les domaines les plus différents : administratif, économique, et notamment linguistique ${ }^{16}$. En effet, les articles 110 et 111 de la Guillelmine, en décrétant l'utilisation du français dans les actes officiels, posent le premier jalon de l'unification linguistique de la France. 
12 Si ces dernières prescriptions sont à juste titre célèbres, ayant ainsi fait l'objet de nombreuses analyses, l'Ordonnance sur fait de la justice de 1539 contient aussi d'autres mesures importantes. Celle-ci, à l'instar d'autres législations criminelles promulguées à cette époque-là en Europe ${ }^{17}$, s'inscrit dans le processus général de constitution et d'affermissement des états nationaux. L'État-machine, qui se dessine dans ces codes de lois, évoque le pouvoir judiciaire et ambitionne le législatif qui en France, à cette époquelà et jusqu'à 1789 , est encore soumis à la Coutume. Il aspire à concentrer dans son appareil administratif l'autorité morale et matérielle de légiférer, de surveiller, de juger et finalement de punir. Dans cette perspective, il réaménage à son profit les sources du droit (en France après Montils-lès-Tours ${ }^{18}$ ). Il se dote en même temps d'une armée, d'une police et d'une bureaucratie à même de contrecarrer les ingérences de l'Église, des seigneuries et des communes. Il esquisse surtout des règles procédurales visant la réduction du pouvoir d'action des personnes privées et la répression du crime en vue du maintien de l'ordre.

13 C'est justement dans ce but qu'agit Poyet, dont le pragmatisme radical et la longue expérience du barreau façonnent l'Ordonnance en tant qu'instrument puissant d'action et de contrôle dans le domaine judiciaire, solidement aux mains de l'autorité monarchique ${ }^{19}$. La vocation fonctionnelle y apparaît déjà dans le préambule, là où le législateur affirme opérer « pour aucunement pourvoir au bien de notre justice, abréviation des procès, et soulagement de nos sujets $»^{20}$. Aussi l'Ordonnance de Villers-Cotterêts, qui n'est pas un code comme d'ailleurs ne le sera pas non plus celle de Saint-Germain-en-Laye (1670), rassemble-t-elle une multitude de sujets disparates dans le dessein d'y mettre un peu d'ordre dans l'intérêt du trône. Comme les contraintes éditoriales ne nous permettent pas d'analyser en détail cet imposant texte de loi, nous nous bornerons à en illustrer la structure d'ensemble, en signalant surtout les éléments novateurs qui seront approuvés par Du Fail.

14 Les cinq premiers articles contiennent des dispositions très restrictives, visant à comprimer les compétences des Officialités diocésaines aux seules actions contre les clercs dans un domaine strictement spirituel.

15 Les articles 6 à 48 sont envisagés en vue d'une brusque accélération des procès. Et ce, non seulement en proscrivant les appels frivoles et autres pratiques dilatoires, les récusations calomnieuses, les allégations des défenseurs en matière de droit et la réception des contumaces comme appelants, mais encore en réduisant les délais des procès et les défauts accordés aux parties et en disposant l'ajournement à personne et à domicile et l'interrogatoire des parties sous serment.

16 Les articles 49 à 85 légifèrent sur différents points substantiels au civil, et notamment à l'endroit des lettres scellées, des bénéfices, des héritages, des créances, des séquestres et des ventes à l'enchère des biens exécutés. Aux articles 50-55, on oblige les paroisses et couvents à tenir des registres fiables des naissances et des sépultures, à remettre chaque année au greffe du baillage ou de la sénéchaussée.

17 Les articles 86 à 138 affichent des dispositions générales portant en particulier sur la procédure civile, entre autres à l'égard des publications d'enquêtes et communications d'inventaires, des condamnations de dommages et intérêts, des exécutions d'arrêts et des appels interjetés contre ceux-ci, des récusations de conseillers exécuteurs, des conduites à tenir de la part des juges, des délibérations à prendre par majorité simple, des absences injustifiées des officiers de cour souveraine, de la fréquence des mercuriales, de 
l'insinuation (c'est-à-dire de l'enregistrement taxé) des donations testamentaires, des contrats signés par les mineurs. Les articles 110-111, nous venons de le rappeler, prescrivent l'utilisation du «langage maternel français » dans la rédaction des actes officiels, en vue de nuire à l'action des cours canoniques s'exprimant en latin ${ }^{21}$, mais aussi dans un but louable de clarté et d'unification linguistique du royaume, qui ne saurait être sans conséquences sur l'écriture littéraire elle aussi.

Les articles 173 à 184 s'appliquent aux officiers judiciaires et en l'occurrence aux notaires et tabellions, sommés de mettre à jour les registres et protocoles des testaments et des contrats, et aux sergents, dont le salaire est fixé avec défense de toute autre entrée supplémentaire, sous peine de perdre leur office.

Les huit derniers articles (185-192) disciplinent des matières inhérentes au droit du travail, en défendant, en fonction du développement du capital manufacturier, les corporations et confréries d'arts et métiers et, qui plus est, les associations et les syndicats de compagnons et ouvriers.

20 Ceci dit par souci d'exhaustivité, le cœur de l'Ordonnance se situe aux articles 139 à 172 qui définissent une réglementation d'ensemble de la procédure criminelle. Du traité d'Esmein à nos jours ${ }^{22}$, le déroulement du procès pénal suivant Villers-Cotterêts a fait l'objet de nombreuses descriptions. Inutile donc de s'y attarder. Il convient en revanche d'en signaler les articles les plus remarquables et novateurs: 139-140, primauté du criminel sur le civil ; 144, engagement ex officio de la poursuite de la part de l'instructeur ; 146, interrogatoire des prévenus de vive voix et en garde à vue; 152, interdiction d'élargissement pour les accusés jugés à l'extraordinaire ; 153, récolement des témoins sous serment et en l'absence des accusés ; 154-155, confrontation sous serment pour les deux parties et présentation des reproches préalablement à la lecture des dépositions ; 159, interrogatoire des témoins à décharge aux frais de l'accusé ; 162, suppression de tout conseil et défense technique pendant les interrogatoires des parties ${ }^{23}$, ayant de surcroit lieu séparément, secrètement et à l'insu des chefs d'imputation; 163-164, exécution de la question extraordinaire (c'est-à-dire de la torture) avec acquittement et réparation faute d'aveu ; 165, équipollence du récolement à la confrontation pour les contumax ; 168-172, interdiction aux gardes des sceaux d'accorder grâces ou rémissions d'aucune sorte aux condamnés, sauf en cas de légitime défense.

21 Tout cela revient à consacrer le rôle principal du juge, dominus incontesté et tout-puissant du procès pénal, arbitre et partie à la fois, détenteur d'une vérité qui n'est pas seulement judiciaire ${ }^{24}$. De la disparité manifeste entre la partie publique et la partie privée, en plus des aboutissements presque physiologiques de la légalité des preuves (à laquelle d'ailleurs ne correspond pas une égale définition normative des peines), découle une suite interminable d'abus et de prévarications. In primis le recours quasiment automatique à la torture (elle aussi dépourvue de toute discipline instrumentale) ayant attiré, depuis des siècles, des critiques sévères et largement motivées ${ }^{25}$.

Ces réserves faites, il faut tout de même reconnaître la valeur historique de l'Ordonnance de 1539, dont les dispositions s'efforçaient - sur le fondement ontologique d'une vérité extrajudiciaire dont le magistrat serait l'interprète et le garant - de porter remède aux désordres et aux débordements de la justice féodale dans un contexte d'insécurité diffuse, provenant de cent cinquante ans de guerres presque continuelles. Aussi, agencée en fonction du dessein centralisateur de la monarchie, restera-t-elle longtemps en vigueur après la disgrâce de son auteur et jusqu'en 1670, quand la Grande Ordonnance promue par Louis XIV, loin de l'adoucir, en alourdira davantage l'orientation totalitaire. C'est 
ainsi que, déjà en décembre 1540, l'Édit sur l'administration de la justice en Normandie ${ }^{26}$, conçu afin d'humilier les prétentions des parlementaires rouennais, ayant refusé l'enregistrement de l'Ordonnance sur le fait de la justice, en aggrave les dispositions punitives. Et ce, notamment à l'égard des crimes commis sur les grands chemins (articles 35-37), de la corruption des officiers de justice (articles 2 et 21) et de la subornation des témoins (article 4).

À la lumière des considérations générales qui viennent d'être exposées sur le développement unitaire de l'Europe continentale en vue de l'adoption de la procédure inquisitoire, il faut aussi se demander si toutes les mesures draconiennes contenues dans l'Ordonnance de 1539 sont dues au chancelier Poyet. Bien que l'action novatrice de celuici fût reconnue et appréciée, la réponse ne saurait qu'être négative. En effet, ainsi qu'en témoigne l'article 167, Villers-Cotterêts, sans annuler « le surplus des ordonnances [...] cidevant faictes sur le faict desdites matières criminelles", s'inspire largement de nombreux précédents juridiques. Entre autres à l'Ordonnance de Blois ${ }^{27}$ (1498) qui avait déjà instauré clairement la distinction entre la voie ordinaire et la voie extraordinaire en fixant les traits saillants de cette dernière : le secret, la coercition, la célérité.

Plus récemment, l'Édit de Valence ${ }^{28}$ sur le fait de la justice en Bretagne (1536) avait créé un précédent des plus notables. Il contient en fait des mesures en avance à plus d'un titre sur le texte de Villers-Cotterêts. Les articles 1 et 2 autorisent la poursuite d'office. Les articles 10 et 11 prévoient l'interrogatoire des prévenus sans l'assistance légale et en garde à vue. C'est toujours en prison ferme que, suivant l'article 15, les confrontations auront lieu entre les témoins et les accusés, dont les reproches préalables ne seront présentés, d'après l'article 16, qu'avant la lecture des dépositions. L'article 21 assigne aux accusés les frais de comparution et l'interrogatoire des témoins à décharge. L'article 28 établit l'équivalence entre récolement et confrontation en cas de contumace. Enfin, l'article 37 défend aux officiers judiciaires de communiquer aux parties toute notice relative à l'information les concernant.

Tout Villers-Cotterêts est déjà là, serait-on tenté de dire, cet édit portant toutefois la signature d'Antoine Du Bourg, prédécesseur de Guillaume Poyet à la chancellerie. Et pourtant, il faut se rappeler qu'en cet instant même le président à mortier du Conseil de Bretagne $^{29}$ était justement le futur rédacteur de l'Ordonnance de Villers-Cotterêts (Angevin comme la plupart des conseillers non originaires de la Cour rennaise). À l'époque, celui-ci était de surcroît chargé de promouvoir une réformation du «Style de Bretagne » qui le normalise aux standards procéduraux français, et auteur à cette fin d'une Pratique judiciaire dont le manuscrit inédit est mentionné par le bibliographe La Croix du Maine ${ }^{30}$.

Or, depuis 1571 et jusqu'en 1586, c'est-à-dire durant la rédaction des Contes et discours, Noël Du Fail siégea, avec la charge de conseiller, dans cette même cour souveraine que Poyet venait de reconstituer. Voici, parmi d'autres qu'on verra par la suite, une première raison à même de motiver les hommages à l'adresse du chancelier, ainsi que les échos récurrents à son œuvre législative parsemés dans le recueil dufailien.

Le souci d'abréviation des procès y est dès lors omniprésent, Polygame et ses compagnons devisant à maintes reprises «des fascheries, importunitez, longueurs, dissimulations, eloignemens et traverses qui se font aux procés » $(C D E, I, 3)$ en vue d'en dilater le cours, alors que le fonctionnement correct de la machine judiciaire demanderait "police et celerité tout ensemble $»(C D E, \mathrm{I}, 100)^{31}$. 

motif tout aussi bien exploité, qui est en outre traité par Du Fail précisément dans l'optique de l'attribution d'un nombre maximal de cas ("privilégiés ", les appelait-on dans l'entourage gallican) au ressort des cours séculières. En fait, c'est toujours l'avis de Du Fail, « un prince souverain n'est juge et magistrat qu'à demy, et est manchot d'une main, s'il ne cognoist, ou son officier, de tous delits indifferemment et absoluement entre toutes personnes, de quelque grade, dignité, qualité et condition qu'elles soyent » (CDE, II, 25-26) $)^{32}$

On a déjà fait référence au blâme dufailien à l'encontre des officiers concussionnaires, fussent-ils de robe longue ou courte, des magistrats de cour souveraine ou d'humbles sergents. Encore plus farouche est l'inimitié qu'il ressent pour les avocats ${ }^{33}$. C'est la raison pour laquelle Polygame se souvient sans aucun regret de l'époque où, Poyet n'y ayant pas encore apporté remède, ils « avoient voix deliberative [...], les procés criminels se juge [a]nt à huis ouverts et en plaine audience, en presence du prisonnier » (CDE, I, 51-52). $C D E$, II, 19), cela est dû « suivant l'ordonnance de l'an 1539 »(CDE, I, 36). C'est à celle-ci que l'on renvoie expressément lorsqu'il est question des modalités des interrogatoires, le juge y étant autorisé, de l'avis de Polygame, à «s'efforce[r], par petites subtilitez et interrogatoires exquis, arracher ainsi une espece de verité d'un prisonnier assez affligé d'ailleurs » (CDE, II, 25). On y fait aussi référence pour ce qui est de la nécessité d' " ouyr les parties en personne et de vive voix » $(C D E, I, 118)$, sous serment de dire la vérité et «sans souffrir que le procureur, vray nourrisson de mensonge, suborne et destourne la religion de sa partie » $(C D E, \mathrm{I}, 36)$. C'est encore conformément "à l'ordonnance qui est requise en tel cas » qu'un juge somme un accusé de reprocher un témoin avant que la confrontation n'ait lieu, « car par cy après n'y serez aucunement receu » (CDE, II, 48-49).

Nous avons déjà mentionné l'adhésion aveugle au penchant répressif qu'inspire l'Ordonnance, la clémence du juge étant pour Du Fail toujours déplorable en cas de " crimes enormes ou commis de guet à pans », quelque " apparence de compassion » fûtelle avancée comme prétexte (CDE, II, 26). L'œuvre novatrice de Poyet est évoquée aussi lorsqu'il s'agit de l'octroi des grâces, un droit régalien «dependant de [1]a seule grandeur» $[C D E, \mathrm{I}, 126]$ du souverain justicier. Elle l'est aussi, plus prosaïquement, à propos d'une bande de témoins centenaires appelés à se prononcer, faute de registres fiables des baptêmes et des sépultures, « sur une question d'héritage » (CDE, II, 189).

Finalement, par l'entremise des péripéties survenues au forgeron Tourtelier ( $C D E$, 126-129), on fait aussi référence à l'existence révolue des corporations d'arts et métiers, abolies par Poyet, ainsi qu'à celle des confréries et abbayes de jeunesse, elles aussi interdites par le chancelier (CDE, II, 181). surtout, comment expliquer, en se posant dans l'optique de l'historiographie positiviste, qu'un hobereau misonéiste comme le seigneur de la Hérissaye puisse rendre un hommage inconditionné au champion de la modernisation bourgeoise et royaliste dans le domaine de la justice ? C'est ce à quoi nous essaierons de répondre en conclusion.

Primo. Du Fail est un aristocrate breton. Et la noblesse de l'ancien duché, en crise de revenus au $\mathrm{XVI}^{\mathrm{e}}$ siècle $^{34}$ et depuis longtemps francisée ${ }^{35}$, se tourne tout au long du processus d'annexion vers le cône de lumière de la monarchie, attendant des institutions 
françaises de généreuses récompenses en contrepartie à son soutien inconditionné à l'œuvre de conquête ${ }^{36}$.

Secundo. Du Fail est un parlementaire breton. Et la Cour rennaise, mi-partie de conseillers résidents et non originaires, est à l'époque le rempart le plus solide de la politique française dans la Péninsule, quitte à devenir le dernier bastion royaliste pendant les troubles de la Ligue $^{37}$.

Il n'y a donc nullement à s'ébahir des louanges réitérées et souvent hyperboliques prodiguées à l'adresse des rois capétiens ${ }^{38}$ et en l'occurrence de ce François I ${ }^{\text {er39 }}$ qui ne commissionna à Poyet la réformation du Style de Bretagne qu'une fois obtenue la mainmise sur le duché.

37 Aussi la carrière professionnelle de Du Fail à l'intérieur de l'administration judiciaire de la Province se fait-elle, d'un bout à l'autre, sous le signe de la fidélité à l'occupant français et, en contrepartie de son loyalisme, sous les meilleurs auspices du parti de la France. Vingt ans après l'achat d'un office vénal auprès du Présidial rennais, il est reçu, grâce à la protection du seigneur de Rohan, à la Cour des Enquêtes du Parlement de Bretagne, avec la charge de conseiller français préservant à titre exceptionnel les honoraires majorés échéant aux non originaires. Après une courte suspension de ses fonctions à cause d'accointances présumées parmi Ceux de la religion - dirigés d'autre part, en Bretagne, par le mentionné Louis de Rohan -, il est réintégré dans sa charge, bénéficiant, de surcroît, d'un avancement de carrière avec sa nomination, en 1579, à la Grande Chambre du Parlement. Six ans après, atteint de la goutte, il demande et obtient, par l'entremise de son mécène, des lettres d'honorariat signées par Henri III impétrant sa mise à la retraite anticipée avec le privilège de "voix et opinion délibérative "; une démarche sans doute contestable, qui est pourtant entérinée, l'année suivante et sous forme de lettres patentes, dans le but de réduire à néant les résistances de la cour contre le calcul de son ancienneté de service à partir de sa désignation au Présidial.

Ces notices biographiques, en soi anodines, acquièrent une toute autre force probante alors qu'on considère combien le profit personnel est susceptible de s'accorder à l'intérêt particulier de l'aristocratie foncière. En effet, comme l'exégèse matérialiste l'a démontré ${ }^{40}$ , le régime absolutiste a une essence dialectique. Il fonctionne en tant qu'institution médiatrice, à la fois progressiste et conservatrice, conciliant, au nom du maintien de l'ordre, les aspirations de la noblesse d'épée, attachée à la sauvegarde de ses privilèges, et celles des couches aisées du Tiers-État souhaitant à leur tour en bénéficier. L'épilogue breton des guerres civiles est à cet égard exemplaire ${ }^{41}$.

39 Les réformes de la justice, se succédant au cours du XVI ${ }^{e}$ siècle, le prouvent d'une façon criante. Dans le domaine normatif, l'unification poursuivie à partir de Montils-lès-Tours n'arrive à abolir entièrement ni la difformité entre les coutumes régionales, ni le dualisme primaire entre pays de droit écrit et de droit coutumier. De même, la subordination progressive aux appareils étatiques de l'activité judiciaire est lourdement infirmée par la persistance, pas seulement nominale, de magistratures concurrentes, la seigneuriale et l'ecclésiastique en l'occurrence, le système de la vénalité introduisant en plus d'autres insidieux éléments de particularisme.

Enfin, si les innovations procédurales contenues dans l'Ordonnance de Villers-Cotterêts seront confirmées et durcies en 1670, ce sera en raison de leur application partielle ou de leur inobservation manifeste, spécialement en ce qui concerne les articles les plus vexatoires pour la partie privée (154 et 162). 
41 perpétue le lien organique entre fief et justice, l'inflexibilité des mesures prévues ne peut que retomber toute entière sur le dos des composantes les plus faibles du corps social, à plus forte raison si elles sont en situation de marginalité voire d'antagonisme. Nous en voulons pour preuve la destinée malheureuse, au cours des XVI ${ }^{e}$ et XVII ${ }^{e}$ siècles, des vagabonds, des sorcières, des Croquants et Va-nu-pieds, des compagnons en grève et des paysans révoltés ; c'est-à-dire le menu et gros gibier des prévôts des maréchaux, sacrifié à la boulimie inquisitoriale des magistrats.

Du Poyet chez Du Fail, donc, mais aussi bien, pourrait-on dire sans égard pour la chronologie, du Du Fail chez Poyet. Et ce, fort aisément; attendu que, du moins avant 1789, ni le blanc ni le bleu ne composent avec le rouge. À Paris comme à Rennes, et partout ailleurs dans le domaine de la couronne.

\section{NOTES}

1. C. Biet, Droit et littérature sous l'Ancien Régime. Le jeu et la valeur de la loi, Paris, Champion, 2002, p. 7.

2. N. Du Fail, Les Baliverneries d'Eutrapel, éd. G. Milin, Paris, Klincksieck, 1969, p. 6.

3. Ibid., p. 3.

4. La biographie de Du Fail a été établie par A. de La Borderie, « Noël Du Fail. Recherches sur sa famille, sa vie, ses œuvres ", dans Bibliothèque de l'École de Chartes, XXXVIII, 1877, p. 572-646 et E. Philipot, La Vie et l'œuvre littéraire de Noël Du Fail gentilhomme breton, Paris, Champion, 1914.

5. C'est ainsi que Du Fail l'appelle dans la lettre-dédicace de ses Memoires recueillis et extraicts des plus notables et solemnels arrests du Parlement de Bretagne [...], Rennes, J. du Cros, 1579.

6. Id., Contes et discours d'Eutrapel, réimpr. par D. Jouaust et C. Hippeau, Paris, Librairie des Bibliophiles 1875, 2 vol. (dorénavant CDE, suivi de l'indication du volume et de la ou des page/s).

7. De l'avis d'E. Philipot « la réduplication me paraît sévir chez du Fail plus que partout ailleurs ; elle prend ici des proportions presque pathologiques ", Essai sur le style et la langue de Noël Du Fail, Paris, Champion, 1914. Voir aussi V. Cordiner, Quod facit ad rem: Du Fail e il discorso esemplare nel Cinquecento francese, Manziana, Vecchiarelli, 2008, p. 230-254.

8. Voir à ce propos les études magistrales de L. Sozzi (La nouvelle française de la Renaissance, Torino, Giappichelli, 1977, 2 vol. et L'Histoire tragique nella seconda metà del Cinquecento francese, Torino, Genesi Ed., 1991), de G.-A. Pérouse (Nouvelles françaises du XVIe siècle. Images de la vie du temps, Genève, Droz, 1977) et de T. Pech, (Conter le crime. Droit et littérature sous la Contre-Réforme: les histoires tragiques (1559-1644), Paris, Champion, 2000).

9. Nous renvoyons, pour ce qui est de l'évolution générique entre les premières épreuves de $\mathrm{Du}$ Fail et son œuvre majeure, à notre étude " "Si les exemples servent de quelque chose, je sais un beau conte à ce propos". Mélanges récréatifs et (surtout) didactiques dans les Contes et discours d'Eutrapel », dans Contes et discours bigarrés, Cahiers V. L. Saulnier, 28, 2011, p. 53-67.

10. «Le premier et plus advantageux titre d'un roy ou prince de son sang est d'estre appellé gentil-homme, la noblesse ayant fait, choisi et esleu tout tel qu'il est, comme son gouverneur et commandant » (CDE, II, 116).

11. Voir CDE, I, 99, 126, 193, 198 ; II, 23, 25-26, 29, 52. 
12. Nous avons consacré à la représentation dufailienne du personnel judiciaire l'étude monographique suivante : " "Il joüoit de son estat comme d'un baston à deux bouts" : portraits de juges dans l'œuvre de Noël Du Fail », dans J.-C. Arnould (dir.), Juges et criminels dans la narration brève du XVI siècle, Publications numériques du CÉRÉdI, "Actes de colloques et journées d'étude", n. 5, 2010, URL : <http://ceredi.labos.univ-rouen.fr/public/?il-jouoit-de-son-estat-comme-dun.html>.

13. La biographie de Du Poyet a été établie par: Boucher d'Argis, Discours préliminaire, dans Ordonnance du Roi François I Ir, donnée à Villers-Cotterêts, au mois d'août 1539, Paris, Le Boucher, 1786, p. i-li ; C. Porée, Un parlementaire sous François I ${ }^{\text {er }}$, Guillaume Poyet (1473-1548), Angers, Germain et Grassin, 1898 ; M. Deubel, Guillaume Poyet avocat et chancelier, Versailles, Girardin, 1900 ; A. Rousselet-Pimont, Poyet Guillaume, dans P. Arabeyre, J.-L. Halpérin et J. Krynen (dir.), Dictionnaire historique des juristes français (XII ${ }^{e}-X X^{e}$ siècle), Paris, PUF, 2007, p. 640-641; Id., Le chancelier et la loi au XVI siècle d'après l'œuvre d'Antoine Duprat, de Guillaume Poyet et de Michel de l'Hospital, Paris, De Boccard, 2005, p. 12-14 (n. 71, 74-75).

14. Cité dans M. Deubel, Guillaume Poyet avocat et chancelier, cit., p. 41.

15. «Tigre altéré de sang [...], vil esclave de la fortune, lâche flatteur, âme de boue capable des plus basses manœuvres", ainsi l'étiquette J.-J. Garnier dans son Histoire de France depuis l'établissement de la monarchie jusqu'au règne de Louis XIV, t. XXV, Paris, Saillant \& Nyon, 1778, p. 260.

16. Voir à ce propos, A. Parrot, "Guillaume Poyet, chancelier de France », dans L'investigateur. Journal de l'Institut Historique, IV, 7, 1867, p. 5-22 et 49-58, p. 57-58.

17. C'est notamment le cas de la Constitutio Criminalis Carolina en Allemagne, de la Nueva Recopilación de las Leyes en Espagne, de l'Ordonnance sur le fait de la justice criminelle aux Pays Bas, des Constitutiones Dominii Mediolanensis et des Nuovi Ordini (Libro quarto) en Italie et, ce qui ne manquera pas de surprendre, des Marian Statutes en Angleterre. Voir E. Dezza, «"Pour pourvoir au bien de notre justice". Legislazioni statali, processo penale e modulo inquisitorio nell'Europa del XVI secolo ", dans Diritto penale XXI secolo, I, 1, 2002, p. 159-195, p. 176-192.

18. Voir, au sujet de cet édit donnant lieu à la compilation des coutumes régionales, J. Bart, Histoire du Droit, Paris, Dalloz, 2000², p. 54-60.

19. Voir A. Marchisiello, "Abréviation des procès": le strategie dell'ordonnance di VillersCotterêts (1539) per la riforma dell'amministrazione della giustizia », dans G. Rossi (dir.), Il Rinascimento giuridico in Francia. Diritto, politica e storia, Roma, Viella, 2008, p. 179-200, p. 189-192.

20. Ordonnance sur le fait de la justice [août 1539], dans Recueil général des anciennes lois françaises depuis l'an 420 jusqu'à la révolution de 1789, par MM. Isambert, Decrusy, Armet, t. XII, Paris, BelinLeprieur \& Verdière, 1828, p. 600-640, p. 600.

21. Ces prescriptions sont étendues, à l'art. 27 de l'Ordonnance (Code Michaud) sur les plaintes des États assemblés..., [janvier 1629], aux actes produits par les Cours de chrétienté : « Tous actes, sentences, conclusions et autres procédures des officialitez et autres jurisdictions ecclésiastiques seront conçus en langage françois, fors pour ceux qui doivent estre renvoyez à Rome, lesquels seront expédiez en latin comme de coutume ", dans Recueil général des anciennes lois françaises depuis l'an 420 jusqu'à la révolution de 1789, cit., t. XVI, p. 223-342, p. 232.

22. Voir A. Esmein, Histoire de la procédure criminelle en France et spécialement de la procédure inquisitoire depuis le XIII ${ }^{e}$ siècle jusqu'à nos jours, Paris, L. Larose et Forcel, 1882, p. 135-158; M. Deubel, Guillaume Poyet avocat et chancelier, cit., p. 43-48 ; E. Dezza, op. cit., p. 135-158 ; M.-H. Renaut, Histoire du droit pénal. Du Xe siècle au XXI siècle, Paris, Ellipses, 2005, p. 55-58.

23. Il était déjà question des procureurs, avocats et autres " tergiversateurs de justice » aux art. 28 et 34 de l'Ordonnance sur la juridiction du grand conseil [juillet 1539], dans Recueil général des anciennes lois françaises depuis l'an 420 jusqu'à la révolution de 1789, cit., t. XII, p. 575-589.

24. Voir J.-M. Carbasse, Histoire du droit pénal et de la justice criminelle, Paris, PUF, 2006², p. 206-208.

25. Voir F. Cordero, Procedura penale, Milano, Giuffré, $1995^{3}$, p. 21-38 et passim. 
26. Édit sur l'administration de la justice en Normandie [déc. 1540], dans Recueil général des anciennes lois françaises depuis l'an 420 jusqu'à la révolution de 1789, cit., t. XII, p. 707-721.

27. Ordonnances rendues en conséquence d'une assemblée de notables à Blois [mars 1498], Ibid., t. XI, p. 323-379.

28. Édit sur le fait de la justice dans le duché de Bretagne [août 1536], chap. 2, Ordonnance criminelle, Ibid., t. XII, p. 515-525.

29. «[...] auquel fut suplanté », comme le dit Du Fail, «le Parlement ordinaire le second jour d'aoust $1554 »(C D E$, II, 51).

30. Voir A. Rousselet-Pimont, Poyet Guillaume, cit., p. 640.

31. Voir aussi $C D E, \mathrm{I}, 12-13,31-32,95-96)$.

32. Voir aussi CDE, I, 53-54, 95-96, 192.

33. Voir CDE, I, 3-4, 8-9, 271 ; II, 67, 283).

34. Voir J. Gallet, La Seigneurie bretonne (1450-1680). L'exemple du Vannetais, préface de R. Mousnier, Paris, Publications de la Sorbonne, 1983.

35. C'est ainsi que, bien avant l'annexion, la haute noblesse du duché s'exprime, surtout à l'écrit, en français ; un choix linguistique que les articles 110 et 111 de Villers-Cotterêts ont sans doute encouragé. Noël Du Fail lui aussi, tout en s'amusant à « far bretone » dans ses ouvrages littéraires (voir A.Schoysman, «Repérer les régionalismes lexicaux: quelques exemples dans les Propos rustiques de Noël Du Fail [1520 env.-1591]», dans Le français préclassique, 8, 2004, p. 97-114), est sans conteste un écrivain et un juriste français.

36. À l'égard de la conduite déloyale de l'aristocratie bretonne, nous renvoyons à B. d'Argentré, Histoire de Bretaigne, $\mathrm{I}^{\mathrm{re}}$ éd. poursuivie et supprimée, s.l., s.d. [1582] et à notre étude «Le due edizioni dell'Histoire de Bretagne di d'Argentré ", dans Micromégas, 70, M. Colesanti et A. M. Scaiola (dir.) Saggi e studi di letteratura francese, Roma, Bulzoni, 2002, p. 173-193.

37. Voir H. Carré, Essai sur le fonctionnement du Parlement de Bretagne après la Ligue (1598-1612) [Paris 1888], Genève, Mégariotis, 1978, p. 18-36.

38. Voir CDE, I, 29, 115, 119-120, 126, 150.

39. Voir CDE, I, 35, 63, 189, 255-256 ; II, 22-23, 35, 203, 220-222.

40. Voir notamment B.F. Porchnev, Les Soulèvements populaires en France de 1623 à 1648, Paris, SEVPEN, 1963.

41. À ce propos, nous renvoyons à l'étude toujours inégalée de C. Vivanti, Lotta politica e pace religiosa in Francia fra Cinque e Seicento, Torino, Einaudi, 1963.

\section{INDEX}

Mots-clés : Du Fail (Noël), Poyet (Guillaume), Droit et Littérature, Ordonnance de VillersCotterêts, Genre narratif bref (XVIe siècle), Mélanges bigarrés, Parlement de Bretagne 